\title{
Frequency-domain measurement of the spin-imbalance lifetime in superconductors
}

\author{
C. H. L. Quay, ${ }^{1, *}$ C. Dutreix, ${ }^{1,2}$ D. Chevallier,,${ }^{3,4}$ C. Bena,,${ }^{1,5}$ and M. Aprili ${ }^{1}$ \\ ${ }^{1}$ Laboratoire de Physique des Solides (CNRS UMR 8502), Bâtiment 510, Université Paris-Sud, \\ Université Paris-Saclay, F-91405 Orsay, France \\ ${ }^{2}$ Radboud University, Institute for Molecules and Materials, Heyendaalseweg 135, 6525AJ Nijmegen, The Netherlands \\ ${ }^{3}$ Department of Physics, University of Basel, Klingelbergstrasse 82, CH-4056 Basel, Switzerland \\ ${ }^{4}$ Instituut-Lorentz, Universiteit Leiden, P.O. Box 9506, NL-2300 RA Leiden, The Netherlands \\ ${ }^{5}$ Institut de Physique Théorique, CEA Saclay, F-91190 Gif-sur-Yvette, France
}

(Received 8 August 2014; revised manuscript received 21 December 2014; published 1 June 2016)

\begin{abstract}
We have measured the lifetime of spin imbalances in the quasiparticle population of a superconductor $\left(\tau_{s}\right)$ in the frequency domain. A time-dependent spin imbalance is created by injecting spin-polarized electrons at finite excitation frequencies into a thin-film mesoscopic superconductor (Al) in an in-plane magnetic field (in the Pauli limit). The time-averaged value of the spin-imbalance signal as a function of excitation frequency $f_{\mathrm{rf}}$ shows a cutoff at $f_{\mathrm{rf}} \approx 1 /\left(2 \pi \tau_{s}\right)$. The spin-imbalance lifetime is relatively constant in the accessible ranges of temperatures, with perhaps a slight increase with increasing magnetic field. Taking into account sample thickness effects, $\tau_{s}$ is consistent with previous measurements and of the order of the electron-electron scattering time $\tau_{e e}$. Our data are qualitatively well described by a theoretical model taking into account all quasiparticle tunneling processes from a normal metal into a superconductor.
\end{abstract}

DOI: 10.1103/PhysRevB.93.220501

Spin-polarized electrons injected into superconductors eventually disappear into the condensate, which is made up of Cooper pairs of electrons of opposite spin. To disappear, the injected electrons-which become quasiparticles in the superconductor-must lose energy, flip their spin, and recombine with quasiparticles of opposite spin to form Cooper pairs. These processes may be sequential or occur in parallel. For example, (1) quasiparticles may undergo elastic or inelastic spin-flip processes, (2) quasiparticles may lose energy without flipping their spin, and (3) low-energy quasiparticles recombining in pairs necessarily lose a quantity of energy equivalent to the superconducting gap, usually in the form of a phonon. The characteristic time scale for these processes-as well as the order in which they occur and any interdependence between them-can shed light on the microscopic properties of the superconductor, including relaxation pathways [1-9] as well as the gap structure and the pairing mechanism in unconventional superconductors [10-13].

Time- and frequency-domain experiments, whether using transport, optical pump-probe, or other techniques, provide the most direct measure of the time scales involved [14-17]. Most of the work in this area has focused on the recombination of quasiparticles, usually with techniques sensitive to the number of quasiparticles and their diminution over time. A quasiparticle population which is larger than that at equilibrium does not, however, exhaust the possible nonequilibrium phenomena: The quasiparticle population can also manifest spin and/or charge imbalances [18-24]. These do not necessarily relax in the same way, nor on the same time scale. Here, we report a frequency-domain measurement of the lifetime of a spin imbalance in the quasiparticle population in a mesoscopic superconductor.

The idea of our experiment is as follows: We inject spinpolarized quasiparticles into a superconductor, in a Zeeman field, at a finite frequency $f_{\mathrm{rf}}=\omega / 2 \pi$ while measuring the

\footnotetext{
*charis.quay@u-psud.fr
}

time average of the nonlocal signal due to the resulting spin imbalance $S(\omega, t)$. We expect a cutoff at $\omega \approx \alpha / \tau_{s}$, with $\tau_{s}$ the spin lifetime of quasiparticles in the superconductor and $\alpha$ a constant; as explicated below, this is visible because of the highly nonlinear current-voltage characteristic of our detector.

Our samples, fabricated with standard electron-beam lithography and evaporation techniques, are thin-film superconducting (S) bars, with a native insulating (I) oxide layer, across which lie normal metal $(\mathrm{N})$ and ferromagnetic (F) electrodes used either as "injectors" or as "detectors." (Fig. 1). In our devices, $\mathrm{S}$ is aluminum $(8.5 \mathrm{~nm}), \mathrm{I}$ is $\mathrm{Al}_{2} \mathrm{O}_{3}$, $\mathrm{F}$ is cobalt (40 nm with an $\mathrm{Al}$ capping layer), and $\mathrm{N}$ is thick aluminum $(100 \mathrm{~nm})$ with a critical magnetic field of $\sim 50 \mathrm{mT}$. (All the data shown were taken with this $\mathrm{Al}$ electrode in the normal state.) A typical device is shown in Fig. 1(a). As in previous experiments, the SIF and NIS junctions have "area resistances," respectively, of $\sim 2$ and $\sim 6 \times 10^{-6} \Omega \mathrm{cm}^{2}$ (corresponding to barrier transparencies of $\sim 4$ and $\sim 1 \times 10^{-5}$ ) and tunneling is the main transport mechanism across the insulator (see the Supplemental Material of Ref. [20]). Measurements were performed at temperatures down to $50 \mathrm{mK}$, in a dilution refrigerator.

We simultaneously perform local and nonlocal transport measurements using standard lock-in techniques: We apply a voltage $V_{\mathrm{dc}}$ across junction $\mathrm{J} 2$, between $\mathrm{N}$ and $\mathrm{S}$, and measure the ("local") current $I$ injected into the superconductor through $\mathrm{J} 2$ and the ("nonlocal") voltages across the other junctions (J1 and J3), which act as detectors. We also measure the local conductance $d I / d V_{\mathrm{dc}}$ and the nonlocal differential signal $d V_{\mathrm{nl}} / d V_{\mathrm{dc}}$. (The lock-in frequency is typically $\sim 10 \mathrm{~Hz}$ and the excitation voltage 10-20 $\mu \mathrm{V}$.) The distance between injection and detection junctions is $\lesssim 1 \mu \mathrm{m}$, well within the spin-imbalance relaxation length in the superconducting state in $\mathrm{Al}$ [21]. In the presence of an in-plane magnetic field $H$ (applied parallel to the nonsuperconducting electrodes), electrons injected into the superconductor create a spin imbalance in its quasiparticle population due to the Zeeman effect [20]. The nonlocal voltage drop $V_{\mathrm{nl}}$ at $\mathrm{J} 3$ is proportional 

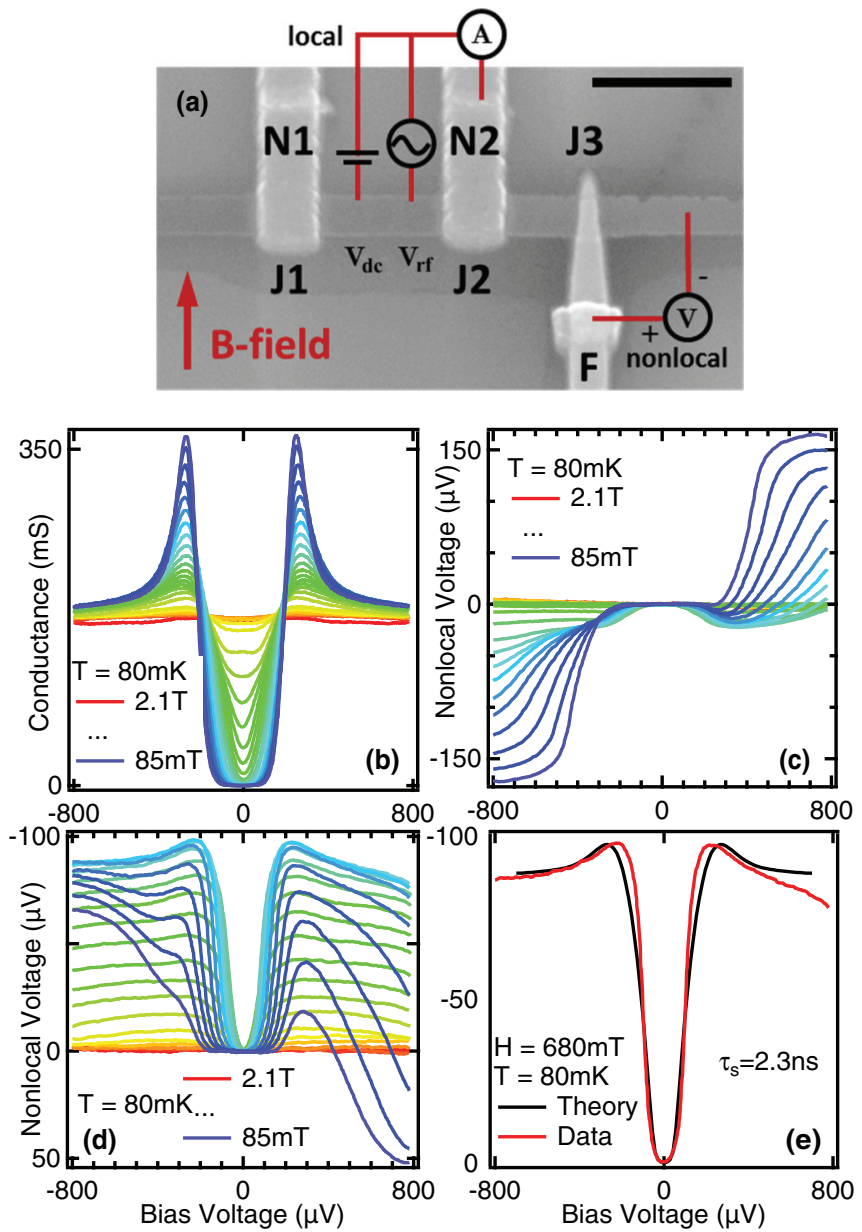

FIG. 1. (a) Scanning electron micrograph of a typical device (scale bar $=1 \mu \mathrm{m}$ ) and schematic drawing of the measurement setup. $\mathrm{S}=$ superconductor $(\sim 8.5 \mathrm{~nm}$ thick $\mathrm{Al}$ film with a native oxide), $\mathrm{N}=$ normal metal $(100 \mathrm{~nm} \mathrm{Al}), \mathrm{F}=$ ferromagnet $(40 \mathrm{~nm} \mathrm{Co}$, with a $4.5 \mathrm{~nm} \mathrm{Al}$ capping layer). The native oxide on $\mathrm{S}$ constitutes a tunnel barrier between it and any other given electrode. Quasiparticles are injected into $S$ across a tunnel barrier by applying a voltage $V_{\mathrm{dc}}$ across $\mathrm{J} 1$ or $\mathrm{J} 2$. These are spin polarized because of the Zeeman field in S. The nonlocal voltage $V_{\mathrm{nl}}$ and differential nonlocal signal $d V_{\mathrm{nl}} / d V_{\mathrm{dc}}$ are measured between $\mathrm{F}$ and $\mathrm{S}$ (at J3) as a function of magnetic field and temperature, as well as a function of the amplitude $V_{\mathrm{rf}}$ and frequency $f_{\text {rf }}=\omega / 2 \pi$ of high-frequency (1-50 MHz) voltages applied to the injection electrode. The local conductance $d I / d V_{\mathrm{dc}}$ is measured simultaneously at the injection electrode. (b) The conductance $d I / d V_{\mathrm{dc}}$ across $\mathrm{J} 2$, which is proportional to the quasiparticle density of states in the superconductor, as a function of $V_{\mathrm{dc}}$ at different magnetic fields. (c) The nonlocal voltage $d V_{\mathrm{nl}}$ measured at $\mathrm{J} 1$ as a function of $V_{\mathrm{dc}}$ at the same fields. (d) The nonlocal voltage $d V_{\mathrm{nl}}$ measured at $\mathrm{J} 3$ as a function of $V_{\mathrm{dc}}$ at the same fields. (e) Theoretical fit to one of the traces in (b), which yields an estimate of $\tau_{s}$ at $H=680 \mathrm{mT}$ of $2.3 \mathrm{~ns}$. We also obtained 1.2, 1.6, and $2.6 \mathrm{~ns}$ for 425 , 510 , and $936 \mathrm{mT}$, respectively. The error is $10 \%-20 \%$ based on the fits and could be larger if uncertainties in the spin-resolved DOS are considered.

to either $\left(\mu_{\mathrm{QP} \uparrow}-\mu_{P}\right)$ or $\left(\mu_{\mathrm{QP} \downarrow}-\mu_{P}\right)$, depending on the relative alignments of the $\mathrm{F}$ magnetization and the magnetic field. Here, $\mu_{\mathrm{QP} \beta}$ is the chemical potential of the spin $\beta$ quasiparticle population and $\mu_{P}$ the Cooper pair chemical potential. We remind the reader that $\mu_{C}=\left(\mu_{\mathrm{QP} \uparrow}+\mu_{\mathrm{QP} \downarrow}\right) / 2$ and $\mu_{S}=\left(\mu_{\mathrm{QP} \uparrow}-\mu_{\mathrm{QP} \downarrow}\right) / 2$ quantify charge and spin accumulation, respectively. The nonlocal voltage drop at $\mathrm{J} 1$ is proportional to $\mu_{C}-\mu_{P}$.

To explore the frequency dependence of the spin imbalance, we add higher-frequency components of amplitude $V_{\text {rf }}$ and frequency $f_{\mathrm{rf}}=500 \mathrm{kHz}-50 \mathrm{MHz}$ to $V_{\mathrm{dc}}$ via a bias tee located next to the device and at low temperature. [See Fig. 1(a) and the Supplemental Material [25].)

Before presenting the experimental data, let us sketch out our main theoretical expectations. We assume that the spin accumulation $S$ in the superconductor satisfies

$$
\frac{d S(t)}{d t}=I_{s}(t)-\frac{S(t)}{\tau_{s}},
$$

where $\tau_{s}$ is the spin relaxation time in the superconductor and $I_{s}$ the spin current.

This equation admits an exact analytical solution:

$$
S(t)=e^{-t / \tau_{s}} \int_{0}^{t} d t^{\prime} I_{s}\left(t^{\prime}\right) e^{t^{\prime} / \tau_{s}} .
$$

We first consider a spin current of the form $I_{S}(t)=I_{\mathrm{dc}}+$ $I_{\mathrm{rf}} e^{i \omega t}$, where $I_{\mathrm{dc}}$ and $I_{\mathrm{rf}}$ are constants, so we then have

$$
S(t)=\tau_{s} I_{\mathrm{dc}}+\frac{\tau_{s} I_{\mathrm{rf}}}{1+\omega^{2} \tau_{s}^{2}} e^{i(\omega t+\phi)}+\text { transient terms, }
$$

with $\phi$ a constant phase. The amplitude of the oscillations in $S(t)$ [and thus $\mu_{s}(t)$ and $V_{\mathrm{nl}}(t)$, the quantity we measure] are frequency dependent and show a Lorentzian cutoff, however, the time-averaged spin accumulation $\langle S(\omega, t)\rangle_{t}$ is frequency independent. This would seem to imply that high-frequency detection is required.

Nevertheless, considering a voltage bias and nonlinear current-voltage characteristics at injector and/or detector junctions, numerical calculations show that there is also a cutoff in $\left\langle V_{\mathrm{nl}}(\omega, t)\right\rangle_{t}$ at $\omega=\alpha / \tau_{s}$ [26]. In our devices, both injection and detection junctions are nonlinear, due to the energy-dependent Bardeen-Cooper-Schrieffer (BCS) quasiparticle density of states (DOS) in the superconductor. Therefore the high-frequency cutoff of Eq. (3) also appears in the dc nonlocal voltage, and a dc measurement of $\tau_{s}$ is possible. According to calculations based on DOS extracted from the measured conductance $d I / d V_{\mathrm{dc}}, \alpha$ can vary from about 0.2 to 0.6 , depending on both $V_{\mathrm{dc}}$ and $H$.

Our theoretical model is described in Ref. [26] and is based on previous work by Zhao and Hershfield [23], which takes into account all quasiparticle tunneling processes at a normalsuperconducting junction, extended to include the Zeeman effect induced by the magnetic field. In contrast to the (similar) model presented in our previous work [20], no assumptions were made about the amplitude of the Zeeman energy or $\mu_{S}$ (which can be up to half the size of the superconducting gap in these measurements).

Turning now to our measurements, we begin by characterizing our device in the absence of high-frequency excitation. Figure 1(b) shows the local conductance $d I / d V_{\mathrm{dc}}$ as a function of bias voltage and magnetic field. We see that, for this device, the superconducting critical field at $\mathrm{J} 2$ is $\sim 1.9 \mathrm{~T}$. Figures 1(c) and 1(d) show the corresponding nonlocal voltage 
$V_{\mathrm{nl}}$ measured at $\mathrm{J} 1$ and $\mathrm{J} 3$, respectively. We remind the reader that, as the injection electrode is normal, the (anti)symmetric part of this signal comes from the spin (charge) imbalance [20]. Note that $V_{\mathrm{nl}}$ due to spin can be almost half the superconducting gap [Fig. 1(d)]. As in our previous work, we see a spin signal which first increases with magnetic field then dies out as the magnetic field approaches its critical value [Fig. 1(d)]. In contrast, the charge signal diminishes with increasing magnetic field [Figs. 1(d) and 1(e)]. Theoretical fits to data at fixed magnetic field such as those shown in Fig. 1(e) allow us to estimate the spin lifetime $\tau_{S}$ at several magnetic fields, yielding 1.2, 1.6, 2.3, and $2.6 \mathrm{~ns}$ for $425,510,680$, and $936 \mathrm{mT}$, respectively. Based only on the fits, the error on these figures is about $10 \%-20 \%$; however, the real value of the error could be larger as it is difficult to theoretically account for orbital depairing effects, due to a small misalignment of the magnetic field with the device plane as well as stray fields from the Co electrode. We emphasize, nevertheless, that our theoretical model is able to reproduce all qualitative features of our data (Figs. 1-4 [26]).

At a fixed magnetic field of $H=680 \mathrm{mT}$ (to obtain a large nonlocal spin signal), we apply a sinusoidal excitation at $1 \mathrm{MHz}$ while sweeping $V_{\mathrm{dc}}$ and varying the rf power. (All rf amplitudes given, unless otherwise stated, are those at the output of the generator.) The results are shown in Fig. 2. The main effect of the oxf excitation on both the local conductance and the nonlocal signal is the phenomenon known as "classical rectification": As sinusoidal signals spend most time at their extrema, each feature in the original trace is "split" by a distance in bias voltage corresponding to the peak-to-peak amplitude of the rf excitation across the injection junction $\mathbf{J} 2$. The splitting of the BCS coherence peaks in the local conductance [Fig. 2(a)] as well as that of the spin-imbalance peaks in the nonlocal conductance [Fig. 2(b)] are well reproduced qualitatively by our theory [Figs. 2(c) and 2(d)]. (We note that the calculated nonlocal signal is very sensitive to even small changes in the spin-resolved DOS, having almost no effect on the calculated conductance. The local conductance, which we measure, is proportional to the total DOS rather than the spin-resolved DOS.) Figure 2 looks the same for all frequencies, modulo an offset in the rf power due to frequency-dependent attenuation in the rf lines. These measurements can thus be used as a calibration of rf power.

Next, we study the frequency-dependent response of our system at constant rf amplitude at the device, using the value of the local conductance at zero bias voltage as a calibration of $\mathrm{rf}$ amplitude. (The $\mathrm{rf}$ amplitude at the device can be more accurately determined from the location of the "rf-split" peaks and is generally $\sim 250 \mu \mathrm{V}$.) Figure 3(a) shows the nonlocal signal as a function of bias voltage at constant $\mathrm{rf}$ amplitude at 1 and $50 \mathrm{MHz}$. For both frequencies, rf-split peaks appear at the same location, but their amplitudes are different: At frequencies which are high compared to $\sim 1 / 2 \pi \tau_{S}$, the classifically rectified peaks have smaller amplitudes than they do at low frequencies. (Whether peak amplitudes increase or decrease with frequency depend on the particular parameters of the system [26].)

To track the frequency evolution of the peak amplitude, we measure the nonlocal signal as a function of rf frequency at the bias voltages indicated by the dashed lines [Figs. 3(c)

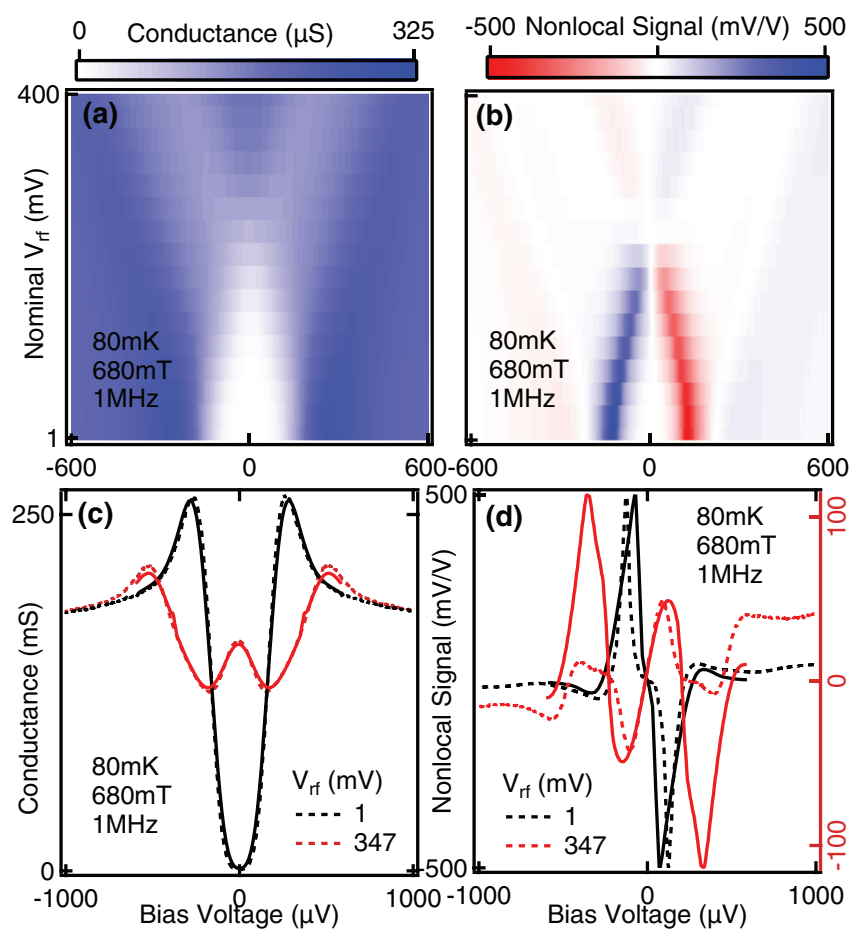

FIG. 2. (a) Measured local conductance $d I / d V_{\mathrm{dc}}$ across $\mathrm{J} 2$ as a function of $V_{\mathrm{rf}}$ at $f_{\mathrm{rf}}=1 \mathrm{MHz}$ and $H=680 \mathrm{mT}$. (b) Measured differential nonlocal signal $d V_{\mathrm{nl}} / d V_{\mathrm{dc}}$ at $\mathrm{J} 3$ as a function of $V_{\mathrm{rf}}$ at $f_{\mathrm{rf}}=1 \mathrm{MHz}$ and $H=680 \mathrm{mT}$. Classical rectification is the dominant rf effect. The $V_{\text {rf }}$ given here is the value at the output of the generator. As noted in the main text, $V_{\mathrm{rf}}$ at the device can be estimated from the classical rectification of features in the $V_{\mathrm{rf}}=0$ trace. (c), (d) Two slices of (a), (b) plotted as dotted lines together with numerical calculations (solid lines) based on the superconducting DOS extracted from the measured local conductance at $V_{\mathrm{rf}}=0$. Small "mismatches" in the conductance can lead to large differences in the nonlocal signal; however, the theory qualitatively agrees with the data.

and 3(d)]. We fit our data to numerical calculations of the peak height to obtain, at $680 \mathrm{mT}$ and $60 \mathrm{mK}, \tau_{s}=6.4 \mathrm{~ns}$ for the inner peaks and $\tau_{s}=3.2 \mathrm{~ns}$ for the outer peaks. The corresponding figures at 425 and $936 \mathrm{mT}$ are 6.4/3.8 and $8 / 8.6 \mathrm{~ns}$ for inner/outer peaks, with a fitting error of $10 \%-20 \%$. As also observed in Ref. [22], our data show no changes in $\tau_{s}$ with increasing temperature up to $600 \mathrm{mK}$ as the quasiparticle population is strongly out of equilibrium [25].

Note that both the inner and outer rf-split peaks originate from the same spin-imbalance peak; however, in the case of the inner (outer), the original peak is "excited" together with quasiparticles of lower (higher) energy. Our results thus suggest that $\tau_{s}$ may depend on the quasiparticle distribution, but this conclusion can only be tentative at this juncture due to the sensitivity of the calculated nonlocal signal to the spin-resolved DOS. Thus, while our experimental techniques open up the possibility of studying the distribution dependence of $\tau_{s}$ (which should give insights into the role of quasiparticlequasiparticle interactions on spin relaxation), further progress on both theoretical and experimental fronts are needed: On the theoretical end, more accurate calculations of the spin-resolved 

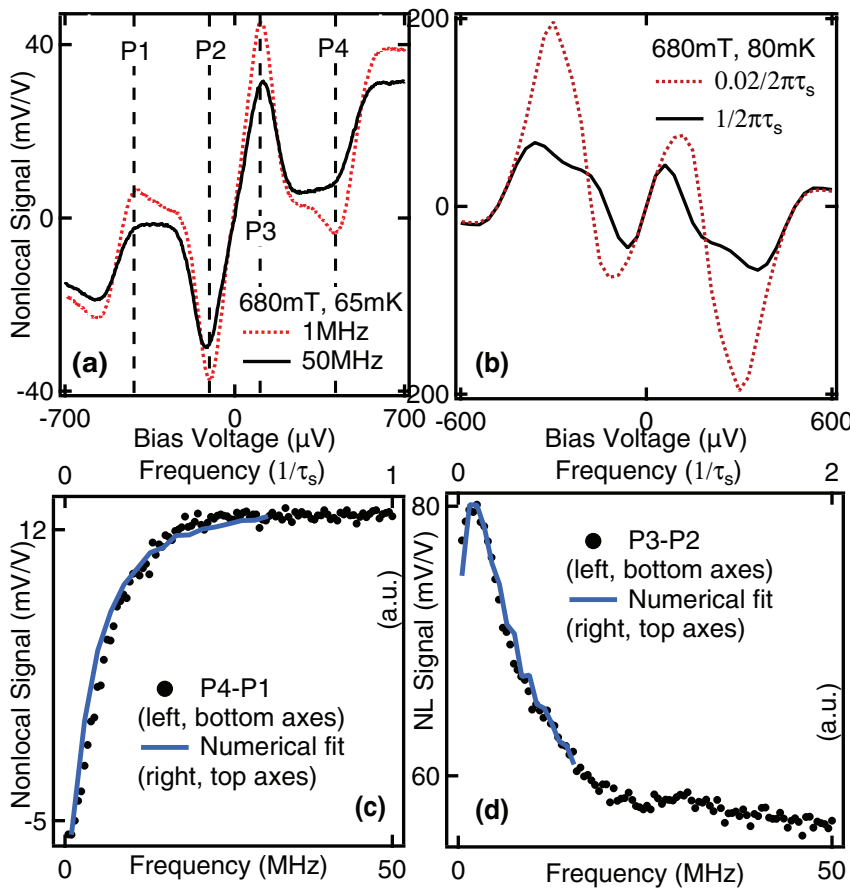

FIG. 3. (a) Differential nonlocal signal $d V_{\mathrm{nl}} / d V_{\mathrm{dc}}$ measured at $\mathrm{J} 3$ with injection at $\mathrm{J} 2$ as a function of $V_{\mathrm{dc}}$ with constant-power excitations (of $\sim 250 \mu \mathrm{V}$ at the device) at $f_{\mathrm{rf}}=1$ and $50 \mathrm{MHz}$. (b) Numerical calculation of $d V_{\mathrm{nl}} / d V_{\mathrm{dc}}$ as a function of $V_{\mathrm{dc}}$ with constant-power excitations at $f_{\mathrm{rf}}=0.02 / 2 \pi \tau_{s}, 1 / 2 \pi \tau_{s}$, based on the superconducting DOS extracted from the measured local conductance at $V_{\mathrm{rf}}=0$. (c), (d) $d V_{\mathrm{nl}} / d V_{\mathrm{dc}}$ at the $V_{\mathrm{dc}}$ values indicated in (a) as a function of $f_{\mathrm{rf}}$. We subtract "opposing" peaks to obtain the antisymmetric part of the signal, which is due to spin. Fits to numerical calculations yield $\tau_{s}=3.2$ and $6.4 \mathrm{~ns}$ with a fitting error of $10 \%-20 \%$. Note that the cutoff does not occur exactly at $1 /\left(2 \pi \tau_{s}\right)$.

DOS could be attempted, while on the experimental end, the (stray) out-of-plane field could be minimized.

The results of measurements similar to those shown in Fig. 3, performed at different fields and at the base temperature of the dilution refrigerator $(\sim 60 \mathrm{mK})$, are shown in Fig. 4 , together with numerical calculations. No significant change in the cutoff frequency (within measurement error) was observed in the range of magnetic fields; however, as mentioned above, the numerical fits suggest a slight rise in $\tau_{s}$ with increasing magnetic field. This rise is smaller than that measured in our previous work on thicker Al samples $(20 \mathrm{~nm}$ versus $8.5 \mathrm{~nm}$ here); this is consistent with results by the Karlsruhe group [22], which also indicates a flatter field dependence for thinner samples.

Finally, the $\tau_{s}$ we obtain both from the fits to the dc data and from the frequency cutoffs are lower than those obtained in our previous work [20]. As also observed by the Karlsruhe group [22], $\tau_{s}$ decreases for thinner films. There could be several physical reasons for this, including increased scattering (lower mean free path) and therefore increased spin-flip scattering [27,28]. Although the increased importance of spin-orbit effects at the surface [28] and finite size effects [29] give the right qualitative thickness dependence
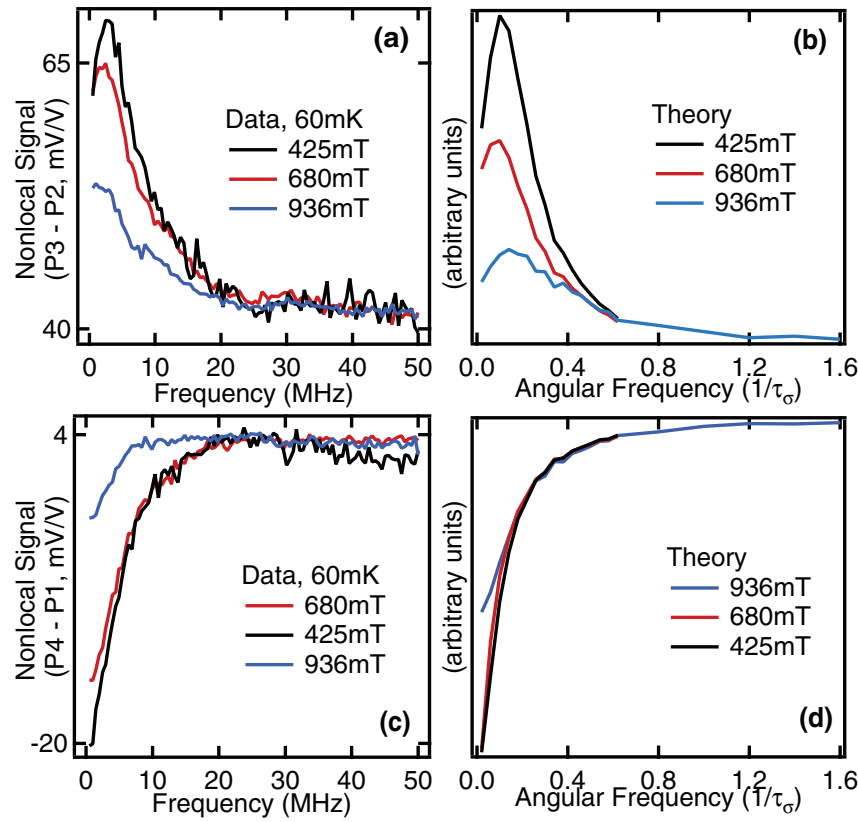

FIG. 4. Differential nonlocal signal $d V_{\mathrm{nl}} / d V_{\mathrm{dc}}$ as a function of rf frequency at different fields for "inner" and "outer" peaks, both (a), (c) experimental data and (b), (d) theory. Both show a decrease in amplitude at high fields. Fits of theory to data suggest a slight rise of $\tau_{s}$ with magnetic field, as well as a difference between inner and outer peaks. (See text.)

for $\tau_{s}$, the spin-orbit scattering measured in $\mathrm{Al}$ thin films of the same thickness is two orders of magnitude smaller $(\approx 50 \mathrm{ps})$ [30]. Both our results and those in Ref. [22] are consistent with $\tau_{s} \approx \tau_{e e}$, where $\tau_{e e}$ is the electron-electron scattering time. Using the expression for enhanced electron-electron scattering time in thin films predicted by Abrahams-AndersonLee-Ramakrishnan [31], we obtain $\tau_{e e} \approx 5$ ns for our samples $\left(R_{\mathrm{sq}}=14 \Omega\right.$ and $T=60 \mathrm{mK}$ ) and $\tau_{e e} \approx 15 \mathrm{~ns}$ for thicker $\mathrm{Al}$ films in Ref. [22], consistent with previous works [32,33]. To verify this estimate, we have measured $\tau_{e e}$ in the frequency domain following the method presented in Ref. [33], which is based on the enhancement of the critical pair-breaking current by microwave radiation-we obtain $\tau_{e e} \approx 3 \mathrm{~ns}$ [25].

Note that the measured cutoffs are independent of the value of the coupling capacitance of the rf line and of the detector's differential resistance at $V_{\mathrm{dc}}=0$ (due to different levels of depairing due to stray fields), thus ruling out detector bandwidth effects. We also checked that the injection of electrons at several times the superconducting gap energy did not significantly affect the shape of the coherence peaks, and the superconducting gap changes by $\approx 2 \%$ at most [25].

In conclusion, we have measured the lifetime of spin imbalances in the quasiparticle population of a superconductor in the frequency domain. This is the most direct measurement to date of this quantity and our technique enables the study of the role of quasiparticle-quasiparticle interactions in spin relaxation. The charge lifetime could in principle be measured in a similar way, at much higher excitation frequencies. Pushing these experiments one step further, one could look at variations in the spin accumulation either in real time or at the excitation frequency. All of these techniques could in principle 
be used to measure spin lifetimes in other superconducting materials in the Pauli limit.

We thank J. Gabelli for helpful discussions on spin dynamics in superconductors, and J. S. Meyer, M. Houzet, and $\mathrm{T}$. Krishtop for the same on effective-temperature- induced spin imbalances. This work was funded by European Research Council Starting Independent Researcher (NANO-GRAPHENE 256965) and Synergy Grants; an ANR Blanc grant (MASH) from the French Agence Nationale de Recherche; and the Netherlands Organization for Scientific Research (NWO/OCW).
[1] J. R. Schrieffer and D. M. Ginsberg, Phys. Rev. Lett. 8, 207 (1962).

[2] A. Rothwarf and B. N. Taylor, Phys. Rev. Lett. 19, 27 (1967).

[3] C. S. Owen and D. J. Scalapino, Phys. Rev. Lett. 28, 1559 (1972).

[4] J.-J. Chang and D. J. Scalapino, J. Low Temp. Phys. 31, 1 (1978).

[5] T. P. Devereaux and D. Belitz, Phys. Rev. B 44, 4587 (1991).

[6] M. Reizer, Phys. Rev. B 61, 7108 (2000).

[7] S. M. Quinlan, D. J. Scalapino, and N. Bulut, Phys. Rev. B 49, 1470 (1994).

[8] Y. Yafet, Phys. Lett. A 98, 287 (1983).

[9] T. Wakamura, N. Hasegawa, K. Ohnishi, Y. Niimi, and Y. C. Otani, Phys. Rev. Lett. 112, 036602 (2014).

[10] R. D. Averitt and A. J. Taylor, J. Phys.: Condens. Matter 14, R1357 (2002).

[11] J. Demsar, B. Podobnik, V. V. Kabanov, T. Wolf, and D. Mihailovic, Phys. Rev. Lett. 82, 4918 (1999).

[12] N. Gedik, J. Orenstein, R. Liang, D. A. Bonn, and W. N. Hardy, Science 300, 1410 (2003).

[13] I. Madan, T. Kurosawa, Y. Toda, M. Oda, T. Mertelj, P. Kusar, and D. Mihailovic, Sci. Rep. 4, 5656 (2014).

[14] M. Johnson, Phys. Rev. Lett. 67, 374 (1991).

[15] G. L. Carr, R. P. S. M. Lobo, J. LaVeigne, D. H. Reitze, and D. B. Tanner, Phys. Rev. Lett. 85, 3001 (2000).

[16] R. Peters and H. Meissner, Phys. Rev. Lett. 30, 965 (1973).

[17] P. Hu, R. C. Dynes, and V. Narayanamurti, Phys. Rev. B 10, 2786 (1974).
[18] J. Clarke, Phys. Rev. Lett. 28, 1363 (1972).

[19] M. Tinkham and J. Clarke, Phys. Rev. Lett. 28, 1366 (1972).

[20] C. H. L. Quay, D. Chevallier, C. Bena, and M. Aprili, Nat. Phys. 9, 84 (2013).

[21] F. Hübler, M. J. Wolf, D. Beckmann, and H. v. Loehneysen, Phys. Rev. Lett. 109, 207001 (2012).

[22] M. J. Wolf, F. Hübler, S. Kolenda, H. v. Loehneysen, and D. Beckmann, Phys. Rev. B 87, 024517 (2013).

[23] H. L. Zhao and S. Hershfield, Phys. Rev. B 52, 3632 (1995).

[24] S. Takahashi, H. Imamura, and S. Maekawa, Phys. Rev. Lett. 82, 3911 (1999).

[25] See Supplemental Material at http://link.aps.org/supplemental/ 10.1103/PhysRevB.93.220501 for details.

[26] D. Chevallier, M. Trif, C. Dutreix, M. Guigou, C. H. L. Quay, M. Aprili, and C. Bena, arXiv:1408.1833.

[27] F. Beuneu and P. Monod, Phys. Rev. B 13, 3424 (1976).

[28] R. Meservey and P. M. Tedrow, Phys. Rep. 238, 173 (1994).

[29] N. H. Long, P. Mavropoulos, S. Heers, B. Zimmermann, Y. Mokrousov, and S. Blügel, Phys. Rev. B 88, 144408 (2013).

[30] C. H. L. Quay, M. Weideneder, Y. Chiffaudel, C. Strunk, and M. Aprili, Nat. Commun. 6, 8660 (2015).

[31] E. Abrahams, P. W. Anderson, P. A. Lee, and T. V. Ramakrishnan, Phys. Rev. B 24, 6783 (1981).

[32] P. Santhanam and D. E. Prober, Phys. Rev. B 29, 3733 (1984).

[33] P. C. van Son, J. Romijn, T. M. Klapwijk, and J. E. Mooij, Phys. Rev. B 29, 1503 (1984). 